Journal of Accident and Emergency Medicine 1995 12, 218-219

CASE REPORT

\title{
Significant pneumothorax complicating a fractured clavicle
}

\author{
R. J. WILLIAMS
}

Department of Orthopaedics, Central Middlesex Hospital, London, UK

\section{SUMMARY}

Pneumothorax has been described as a complication of a fractured clavicle only three times. It is an important and potentially serious complication. This case report describes a fractured clavicle complicated by a significant pneumothorax which required chest drain insertion.

Keywords: chest drain, clavicle, fracture, pneumothorax.

\section{CASE REPORT}

A 30-year-old female patient presented to the accident and emergency (A\&E) department having fallen off her bicycle. She complained of a painful left shoulder, poorly localized left-sided pleuritic chest pain and a painful right knee. She had not sustained any head injury and had no past medical history of note, including no history of previous pneumothorax.

On examination she was alert, cardiovascularly stable, in no respiratory distress, not clinically hypoxic, and chest examination was normal. She had a clinical fracture of the left clavicle with pain, tenderness and obvious swelling over the anterior shoulder. Some soft tissue injuries were noted over the left anterior chest wall at the level of the second and third rib, but there was no clinical rib fracture. There was palpable surgical emphysema in the left neck, but the neck veins were not engorged, no expanding haematoma was noted and neurovascular examination of the left arm was normal. Further examination was normal, apart from soft tissue injuries around the right knee.

Radiographs showed a completely displaced, oblique fracture of the middle third of the left clavicle, surgical emphysema in both sides of the neck and a $10 \%$ pneumothorax on the left. No rib fractures or other bony injuries were found. In view of the complete absence of respiratory symptoms it was decided not to insert a chest drain.

The patient was admitted for observation and remained stable. However, the following day a repeat chest radiograph (Fig. 1) revealed a $70 \%$ pneumothorax with marked mediastinal displacement to the right. Despite the radiographic appearance the patient remained well with no signs of respiratory impairment. A 16-gauge apical chest drain was inserted under local anaesthetic. After 5 days the lung had expanded completely and the drain was removed. The fracture was treated conservatively in a sling and the patient was allowed to return home. Regular follow-up chest radiographs showed no recurrence of the pneumothorax, and at 6 weeks the clavicle fracture had clinically united.

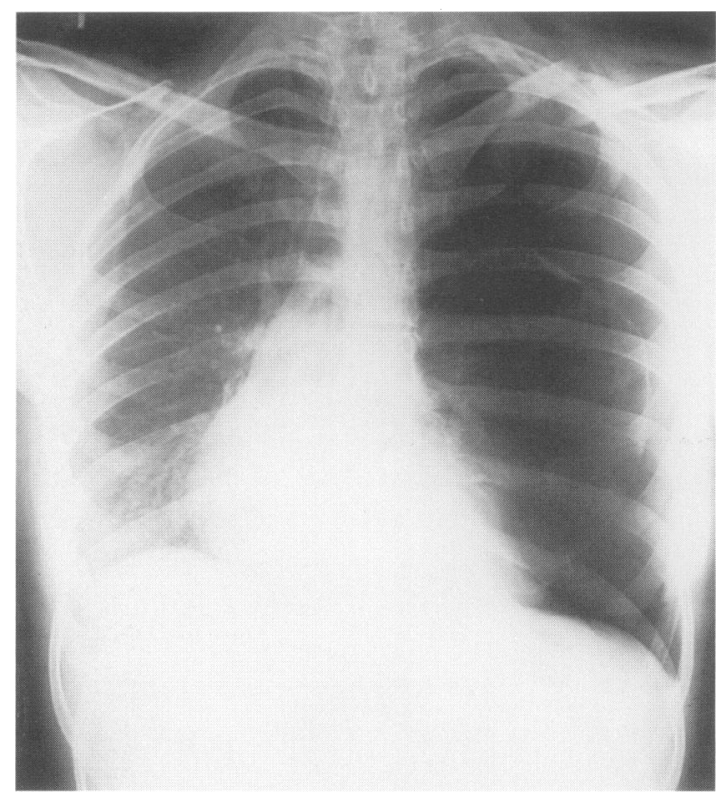

Fig. 1. Chest radiograph taken 1 day after the injury, showing fractured clavicle and pneumothorax. 
Significant

pneumothorax complicating a fractured davicle

\section{DISCUSSION}

Fractures of the clavicle are common. The mechanism of injury is usually due to a fall on an outstretched hand, but can also be caused by direct trauma to the shoulder. Yates ${ }^{1}$ suggested that complications, including pneumothorax, following this injury were more common when due to direct trauma of the shoulder. In the case described here the patient was uncertain exactly how she fell, but the anterior chest tenderness may suggest a direct injury.

Malunion with slight residual deformity is common. However, more serious neurovascular complications are well recognized,,$^{1-3}$ and should always be excluded. Of historical note, the death of Sir Robert Peel followed a fractured clavicle with an expanding haematoma caused by puncture of the subclavian vein. ${ }^{1,4}$ Pneumothorax has been reported as a complication of this injury in the past, but only on three occasions ${ }^{1,4,5}$ and never involving such an extensive lesion. In this respect, clavicular fractures differ from rib and scapular fractures, normally due to direct trauma, which are well recognized causes of pneumothorax.

In the case reported here, no respiratory impairment was noted at any time, but the radiograph taken on the second day shows a significant pneumothorax, i.e. where the mediastinum is displaced to the contralateral side by continuous positive intrapleural pressure. If this had occurred in an elderly patient or a patient with pre-existing lung disease, significant hypoxia may have resulted, requiring emergency treatment of the pneumothorax by chest drain insertion.

Although clavicle fractures are common injuries, rarely requiring more than conservative treatment on an out-patient basis, pneumothorax should be considered as a potential complication and excluded by appropriate examination and radiographs.

\section{REFERENCES}

1. Yates D.W. (1976) Complications of fractures of the clavicle. Injury 7, 189-193.

2. Miller D.S. \& Boswick J.A. (1969) Lesions of the brachial plexus associated with fractures of the clavicle. Clinical Orthopaedics and Related Research 64, 144-149.

3. Howard P.M. \& Shafer S.J. (1965) Injuries to the clavicle with neurovascular complications. Journal of Bone and Joint Surgery 47A, 1335-1346.

4. Correspondence (1952) British Medical Journal, 2, 666.

5. Dugdale T.W. \& Fulkerson J.P. (1987) Pneumothorax complicating a closed fracture of the clavicle. Clinical Orthopaedics and Related Research 221, 312-214. 